\title{
Measurements and Calculations of Halfraum Radiation Drives at the Omega Laser
}

S. A. MacLaren, C. A. Back, J. H. Hammer

January 10, 2005

Radiative Properties of Hot Dense Matter Santa Barbara, CA, United States

November 1, 2004 through November 5, 2004 
This document was prepared as an account of work sponsored by an agency of the United States Government. Neither the United States Government nor the University of California nor any of their employees, makes any warranty, express or implied, or assumes any legal liability or responsibility for the accuracy, completeness, or usefulness of any information, apparatus, product, or process disclosed, or represents that its use would not infringe privately owned rights. Reference herein to any specific commercial product, process, or service by trade name, trademark, manufacturer, or otherwise, does not necessarily constitute or imply its endorsement, recommendation, or favoring by the United States Government or the University of California. The views and opinions of authors expressed herein do not necessarily state or reflect those of the United States Government or the University of California, and shall not be used for advertising or product endorsement purposes.

This work was performed under the auspices of the U. S. Department of Energy by University of California, Lawrence Livermore National Laboratory under Contract W-7405-Eng-48. 


\section{Title}

Measurements and calculations of halfraum radiation drives at the Omega laser

\section{Authors}

Stephan A. MacLaren, Christina A. Back, James H. Hammer

\section{Affiliations}

Lawrence Livermore National Laboratory

\section{Abstract}

Thin walled gold halfraums are a common choice for producing $\mathrm{x}$-ray drives in experiments at high-power laser facilities. At the Omega Laser, we use $10 \mathrm{~kJ}$ of laser energy in a two-pulse sequence to generate halfraum drive temperatures of 160-190 eV for $\sim 3 \mathrm{~ns}$. This type of drive is well characterized and reproducible, with characterization of the drive radiation temperature typically performed using the Dante diagnostic. Additionally, calibrated Photoconductive Diamond Detectors (PCDs) are used to measure the drive when it is desirable to utilize the Dante elsewhere in the experiment. Measurements of halfraum drives from both Dante and PCDs are compared with calculations, with good agreement. This agreement lends the calculations a predictive capability in designing further experiments utilizing halfraum drives.

\section{Keywords}

Halfraum, x-ray drive, Dante, PCD, Omega

\section{Introduction}

It has long been recognized that laser heated gold hohlraums, of the type included in designs for indirect drive Inertial Confinement Fusion (ICF) targets, are also well suited to a wide range of other high energy density physics applications [1]. At the Omega Laboratory for Laser Energetics, half-hohlraums, or halfraums, are driven from one side through a laser entrance hole (LEH), and take advantage of the same benefits of 
conversion efficiency, symmetry, and reproducibility as hohlraums. They typically use less than half of the available beams to drive a target package at the closed end, opposite the LEH. The laser drive configuration used for the experiments described in this paper is illustrated in Figure 1.

Although the left-right symmetry of the full hohlraum is absent, the pattern of $x$ ray illumination on the back wall of the halfraum is well approximated as axisymmetric. This allows for easier modeling of experimental designs based on the halfraum platform. The simulation used to model the drive in these halfraum experiments will be discussed in Section 4, and the output from such calculations will be compared with measurements of the halfraum drive energy in Section 5 .

Halfraum drive measurements can be made using one of several calibrated diamond Photoconductive Diodes (PCDs) from various locations at Omega. The signals from the PCDs are calibrated against an absolute measurement of soft x-ray power from the Dante diagnostic. The calibration procedure is detailed in Section 3, and PCD data will be compared to the simulation results in Section 5. When the PCDs are used to measure the halfraum drive, the Dante diagnostic is available for other views of the experiment, where spectral information may be of greater interest.

\section{Description of Halfraum Experiments}

The laser drive for each of the experiments discussed consists of square pulses in two cones, designed to keep the radiation temperature inside the halfraum at a near constant value for a longer time than a single square pulse. The first pulse consists of 15 beams in cones at angles between 48 and 62 degrees from the normal to the LEH; these beams are the darker shaded outer beams in the geometry shown in Figure 1. The second 
pulse is formed from a cone of six beams at 23 degrees from the normal, shown as the lighter shaded inner beams in the Figure. The pulses are 1.2 and $1.7 \mathrm{~ns}$ in duration for the scale 1 and scale 1.4 targets, respectively.

The halfraum targets shot for these drive measurements are Nova scale 1(scale $1.4)$, or $1.6 \mathrm{~mm}(2.24 \mathrm{~mm})$ inner diameter and $1.28 \mathrm{~mm}(1.79 \mathrm{~mm})$ in length with $10 \square \mathrm{m}$ thick Au walls. Each uses a 75\% laser entrance hole (LEH). They contain two kinds of materials at the closed end, either a $9 \square \mathrm{m}$ thick $\mathrm{Al}$ foil or a $100 \square \mathrm{m}$ thick $\mathrm{Ta}_{2} \mathrm{O}_{5}$ foam.

Measurements of the x-ray drive flux are made through the LEH with two types of diagnostics. The Dante diagnostic consists of an array of absolutely calibrated filtered diodes that employ $\mathrm{Al}, \mathrm{Cr}$, and $\mathrm{Ni}$ photocathodes [2]. In addition, thin filters and mirrors are positioned in front of the cathodes to further define the spectral response of the channels. The resulting spectral coverage ranges from $50 \mathrm{eV}$ to $2600 \mathrm{eV}$ in 12 channels, as shown in Figure 2. The signal from the highest energy channel is used to compensate for the high-energy tails in the responses of channels 5-8. The combination of the diodes with the associated $5 \mathrm{GHz}$ bandwidth oscilloscopes results in a system response on the order of $200 \mathrm{ps}[3]$.

Photoconductive Detectors (PCDs) are also used to measure the radiation temperature of the drive. These detectors consist of $1 \mathrm{~mm}$ cubes of natural type IIa (insulating) diamond crystal biased to $800 \mathrm{~V}$ and become conducting when exposed to an x-ray flux [4]. Provided the carrier density in the diamond produced by the incident $x$ rays is within the desired range, the PCD will produce a linear, flat response with a time response less than $100 \mathrm{ps}$ [5]. A pinhole array is typically placed in front of the PCD to create multiple, overlapping images of the target on the diamond surface while ensuring 
the carrier density is kept from saturation. The presence of several of these detectors at the Omega facility allows for increased flexibility in experimental diagnosis, including use of a PCD to measure the drive temperature, freeing the Dante diagnostic for other views.

The Dante data is spectrally resolved into the filtered channels, allowing an absolute measurement of x-ray power per solid angle to be extracted. A trial blackbody spectrum is convolved with the responses of the channel photocathode, filter, and mirror combinations to calculate estimates of the channel voltages. The spectrum is iterated to fit the measured voltages, and the result is integrated to find the measured power. The PCD signal is proportional to the total x-ray flux onto the crystal surface with no spectral resolution. This voltage is converted to a power measurement by calibrating each PCD in situ with the Dante, resulting in a single calibration constant for each PCD. The PCD power measurement can also provide an equivalent black body radiation temperature by equating the flux to $\square \mathrm{T}^{4}$, where $\square$ is the Setfan-Boltzmann constant.

\section{Details of the PCD Calibration Procedure}

To obtain the calibration constant mentioned above, a signal from the PCD in question must be compared to a Dante measurement for a similar view of the target. This is done in-situ at Omega using a set of five beams onto a simple gold disk target. Due to various factors external to the diamond that may affect the calibration such as detector alignment and positioning, coatings on the diamond, and detector cabling, the calibration is checked each shot day. The target is oriented such that several PCDs and the Dante view the disk at approximately the same angle to the normal. The Dante typically measures a peak flux of 150 to $160 \mathrm{GW} / \mathrm{sr}$ for a one ns square pulse on these targets. 
The current conducted through the PCD during exposure to $\mathrm{x}$-rays from the target is measured as a voltage drop across the diode. This signal is corrected for the time response of the detector system and then compared to the flux measured by the Dante for the same shot, producing a calibration constant in units of $\mathrm{GW} / \mathrm{sr} / \mathrm{volt}$. The calibration is applied to the PCD data from the other shots of the day yielding flux and blackbodyequivalent temperature measurements.

The gold calibration targets radiate approximately one-fourth as much flux as a typical hohlraum or halfraum LEH. It was discovered during the early measurements of these disks that the PCD response was adversely affected by over-filtering of the detector surface using pinhole arrays that did not allow the carrier density within the surface to reach an acceptable level. This resulted in signals from the PCDs whose temporal shapes did not match that of the Dante flux measurement, and calibration values that were not consistent from shot-day to shot-day. Adjusting the pinhole size and spacing to increase the flux onto the detector while keeping the carrier density below saturation vastly improved the detector response for these calibration disk targets. Not only did the PCD signal reproduce the Dante's shape, the value of the calibration constant stabilized and remained consistent over a period of many months. Figure 3 shows calibration measurements from May 2003 and August 2004 for the Dante and H11 PCD, with Dante flux in units of GW/sr on the left axis, and PCD signal in volts on the right. The conversion from volts to flux remains essentially constant for that period.

\section{Computational Model of the Halfraum Drives} Integrated simulations of halfraum drive experiments at Omega are performed

using the radiation hydrodynamics code LASNEX [6]. The simulations use as input the 
actual laser power versus time from the Omega shot database for each cone. In converting from a 3-D to a 2-D geometry, a choice must be made as to how to model the laser beam geometry. In these simulations, the real focusing angles of the beams are preserved, rather than the beam spot intensity at the halfraum wall, as this gives better agreement with the measurements.

In the regions of the simulation expected to be in local thermodynamic equilibrium (LTE), the code uses the supertransition array (STA) opacity model $[7,8]$. The inner surface of the gold halfraum where the laser energy is deposited, however, does not remain in LTE, and an average atom cross section model (XSN) [9] is used for the gold opacity. The simulations are run with two options for the gold opacity. The first method uses XSN exclusively in the non-LTE region, but tunes the wall losses to match those of STA in gold. The second approach has the code automatically transition the gold opacities from those calculated by XSN to those calculated by STA in zones where the electron temperature is below $300 \mathrm{eV}$. Comparisons of these approaches are presented in the next section.

Output from the simulations is post-processed to create synthetic diagnostic measurements for better comparison with experimental data. Rotating the simulation about the axis of symmetry and projecting the radiated x-ray power out from the LEH, taking into account the angle of view of the diagnostic, creates a synthetic measurement of x-ray flux. The flux is then spectrally integrated for comparison with the time resolved PCD or Dante measurements. The synthetic diagnostic flux can also be processed through the different Dante channel response functions for direct comparison with individual channel voltage signals. 


\section{Comparison of calculations with experimental data}

A number of different diagnostic configurations are employed to make the drive measurements presented in this section. Each of the diagnostic angles listed here are measured from the normal to the halfraum LEH. Most of the Omega shots used the P11 PCD to measure the drive at $37.4^{\circ}$. One configuration used the Dante at $41.8^{\circ}$ to measure a scale 1 and a scale 1.4 halfraum with an Al back wall. Finally, one shot employed both Dante and the $\mathrm{H} 11 \mathrm{PCD}$ to view the drive at $70.5^{\circ}$ and $49.0^{\circ}$ respectively.

Figure 4 shows a comparison of the P11 PCD measurement $\left(37.4^{\circ}\right)$ with calculations for a scale 1.4 halfraum, and Figure 5 shows a similar comparison for the scale 1.4 shot with the Dante measuring the flux from $41.8^{\circ}$. The measured data points, indicated with diamond markers, also include the $20 \%$ error bar that is characteristic of the Dante diagnostic. Because it is calibrated to Dante, the same error bar is applied to PCD measurement as well. The figures make it clear that the combination STA/XSN opacity model does a much better job of matching the data during the first (15 beam) laser pulse.

It must also be pointed out that in all cases, the synthetic diagnostic flux from the calculation drops faster than the measured flux once the first set of drive beams turns off. This is either due to errors in the modeling of the energy deposition from the second set of beams, or perhaps simulation of the cooling LEH plasma. In some cases, the calculation shows a spike in flux from the LEH, above the measured value, just prior to the final decay. This is likely due to on-axis plasma stagnation that is enhanced by the axisymmetric nature of the calculation. 3-D calculations are planned to investigate this possibility. 
Further insight into the discrepancy between the two opacity models can be gained by comparing synthetic Dante channel voltages with the measured signals. Figure 6 is such a comparison from the $41.8^{\circ}$ Dante measurement above, showing the channel 4 voltage and the synthetic counterpart from each of the calculations. Figure 7 illustrates the same curves for Dante channel 6. The channel 4 response is centered around $0.7 \mathrm{keV}$, while the response for channel 6 is near $1.0 \mathrm{keV}$. These plots show that the XSN only calculation does a particularly poor job of matching the lower x-ray energy signal. The same level of disagreement for the XSN only model is seen for comparisons of channels $1-3$.

Another method of parsing the Dante data is to separate the soft $(<2 \mathrm{keV})$ and hard $(>2 \mathrm{keV})$ components of the $\mathrm{x}$-ray flux for comparison with calculation. Figures 8 and 9 do this for the scale 1.0 and scale 1.4 Al-backed halfraums, respectively. The hotter scale 1.0 target radiates a larger fraction of the power through the LEH as hard xrays and clearly demonstrates the disagreement between the calculation and the measurement for the harder spectrum. Alternately, a plot of the equivalent blackbody temperatures for these two shots is shown in Figure 10. Here, the model does a reasonable job of modeling the different radiation temperatures resulting from changing the size of the drive cavity.

Finally, the shot configuration allowing simultaneous measurement by Dante and a PCD offers the opportunity to verify the reliability of the PCD measurement and look at how well the model tracks the axial temperature gradient inside the halfraum. The effective blackbody temperatures for the two diagnostics are shown in Figure 11. The H11 PCD used for this measurement had been calibrated to Dante using a gold disk shot 
that day. Both the $49.0^{\circ}$ view of the PCD and the $70.5^{\circ}$ view of the Dante are well matched by the synthetic diagnostic calculations.

\section{Conclusions}

Halfraums at Omega have been used to generate reproducible, axi-symmetric drives that are readily characterized. Both the Dante and the PCD soft X-ray diagnostic have been used successfully at Omega to characterize halfraum drives. An accurately calibrated PCD is a suitable substitute for a Dante drive measurement, allowing flexibility in diagnostic setup and beam configuration at Omega. When simulating the halfraum drive, the combination STA/XSN opacity model yields the best results when compared with measurements. Good agreement between these 2D drive calculations and data provides evidence that the experimental drive is adequately symmetrized, a predictive capability when designing halfraum drives, and confidence that simulations of experiments using a halfraum to drive a package receive the correct "input".

\section{References}

[1] Rosen MD. The science applications of the high-energy density plasmas created on the Nova laser. Phys Plasmas 1996; 3:1803-1812.

[2] Kornblum HN, Kauffman RL, Smith JA. Measurement of 0.1-3-keV x rays from laser plasmas. Rev Sci Instrum 1986; 57:2179-2181.

[3] Decker C, Turner RE, Landen OL, Suter LJ, Amendt P, Kornblum HN, Hammel BA, Murphy TJ, Wallace J, Delamater ND, Gobby P, Hauer AA, Magelssen GR, Oertel JA, Knauer J, Marshall FJ, Bradley D, Seka W, Soures JM. Hohlraum radiation drive measurements on the Omega laser. Phys Rev Lett 1997; 79:1491-1494.

[4] Kania DR, Pan LS, Bell P, Landen, OL. Absolute x-ray power measurements with subnanosecond time resolution using type IIa diamond photoconductors. J Appl Phys 1990; 68:124-130.

[5] Pan LS, Kania DR, Pianetta P, Landen OL. Carrier density dependent photoconductivity in diamond. Appl Phys Lett 1990; 57:623-625.

[6] Zimmerman GB, Kruer, WL. Numerical simulation of laser-initiated fusion. Comments Plasma Phys Controlled Fusion 1975; 2:51-61.

[7] Bar-Shalom A, Oreg J, Boldstein WH, Shvarts D, Zigler A. Super-transition-arrays: A model for the spectral analysis of hot, dense plasma. Phys Rev A 1989; 40:3183-3193. 
[8] Klapisch M, Bar-Shalom A, Oreg J, Colombant D. Recent developments in atomic physics for the simulation of hot plasmas. Phys Plasmas 2001; 8:1817-1828.

[9] Post DE, Jensen RV, Tarter CB, Grasberger WH, Lokke WA. Steady-state radiative cooling rates for low-density, high-temperature plasmas. At Data Nucl Data Tables 1977; 20:397-439.

\section{Figure Captions}

Figure 1: The 21-beam drive used for halfraums at the Omega laser. The outer 15 beams make up the first half of the drive pulse, followed by the inner 6 beams.

Figure 2: (Normalized) responses of the 12 Dante channels

Figure 3: PCD calibration measurements taken 15 months apart, demonstrating a consistent calibration constant of $3.5 \mathrm{GW} / \mathrm{sr} / \mathrm{V}$ for the H11 PCD

Figure 4: P11 PCD measurement of scale 1.4 halfraum drive flux, compared to calculations

Figure 5: Dante measurement of scale 1.4 halfraum drive flux, compared to calculations Figure 6: Voltage signal from Dante channel 4 for scale 1.4 halfraum, compared to synthetic channel 4 Dante voltages

Figure 7: Voltage signal from Dante channel 6 for scale 1.4 halfraum, compared to synthetic channel 6 Dante voltages

Figure 8: Soft $(<2 \mathrm{keV})$ and hard $(>2 \mathrm{keV})$ components of the x-ray flux from a scale 1.0 halfraum

Figure 9: Soft $(<2 \mathrm{keV})$ and hard $(>2 \mathrm{keV})$ components of the $\mathrm{x}$-ray flux from a scale 1.4 halfraum

Figure 10: Equivalent blackbody temperatures for Dante measurements of scale 1.0 and 1.4 halfraums, compared to calculations

Figure 11: Equivalent blackbody temperatures for simultaneous Dante and PCD measurements of the same halfraum drive 


$$
\text { 䊁 }
$$




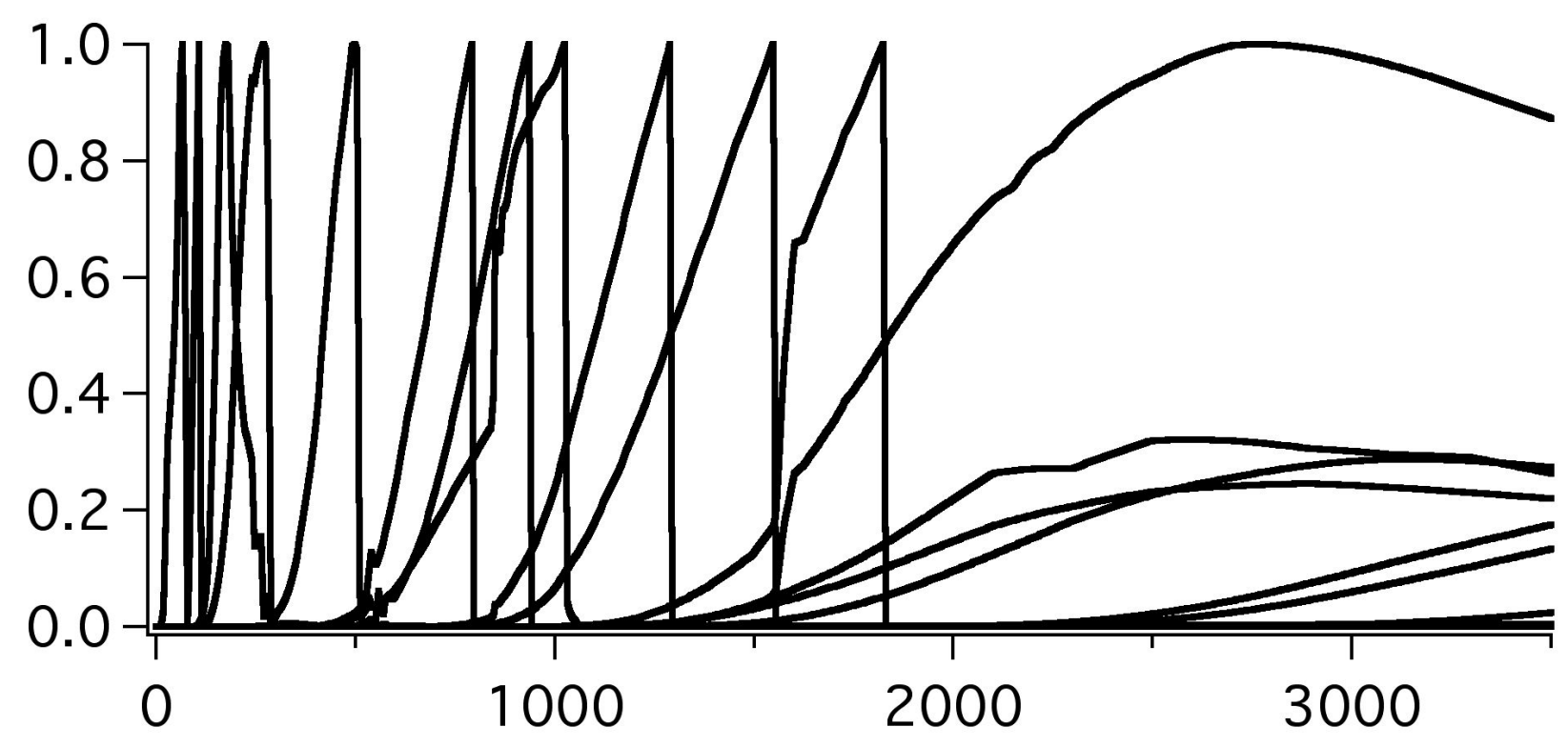




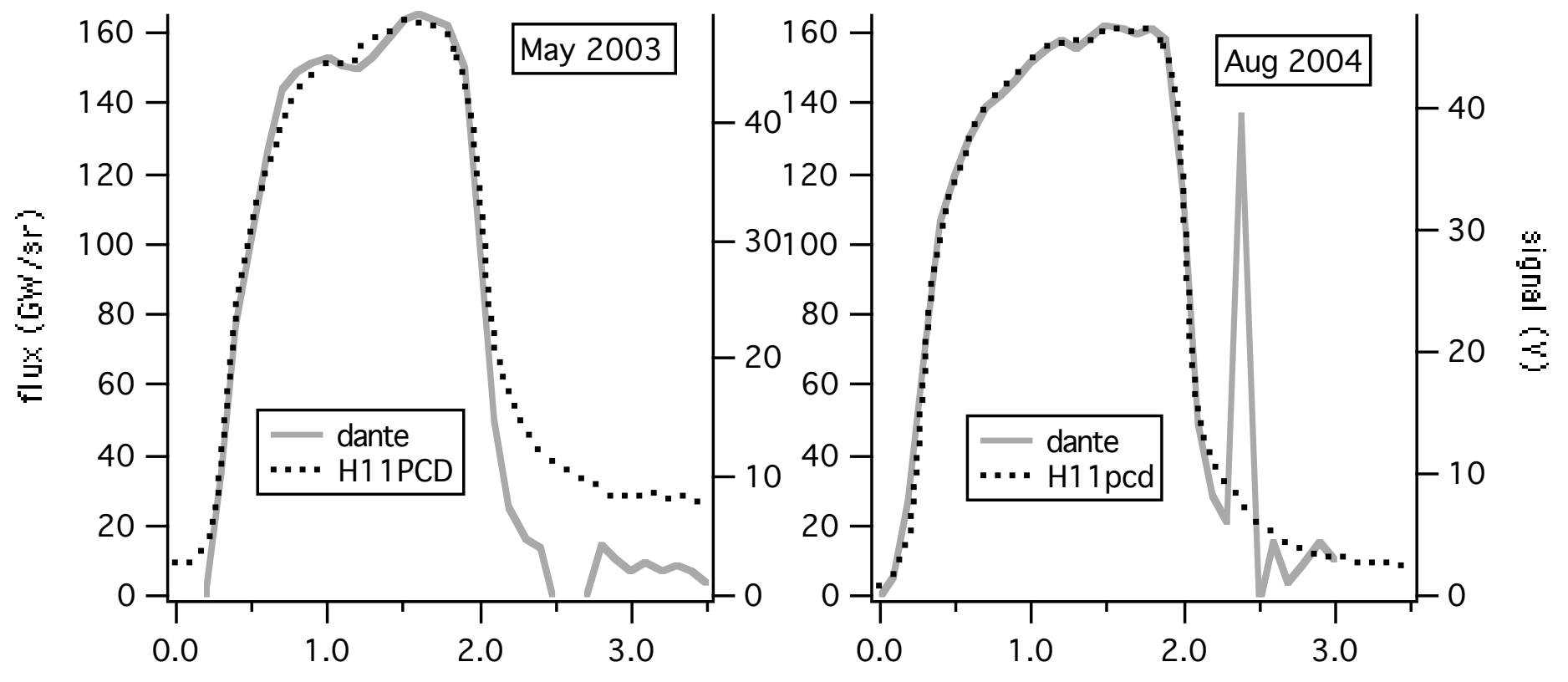




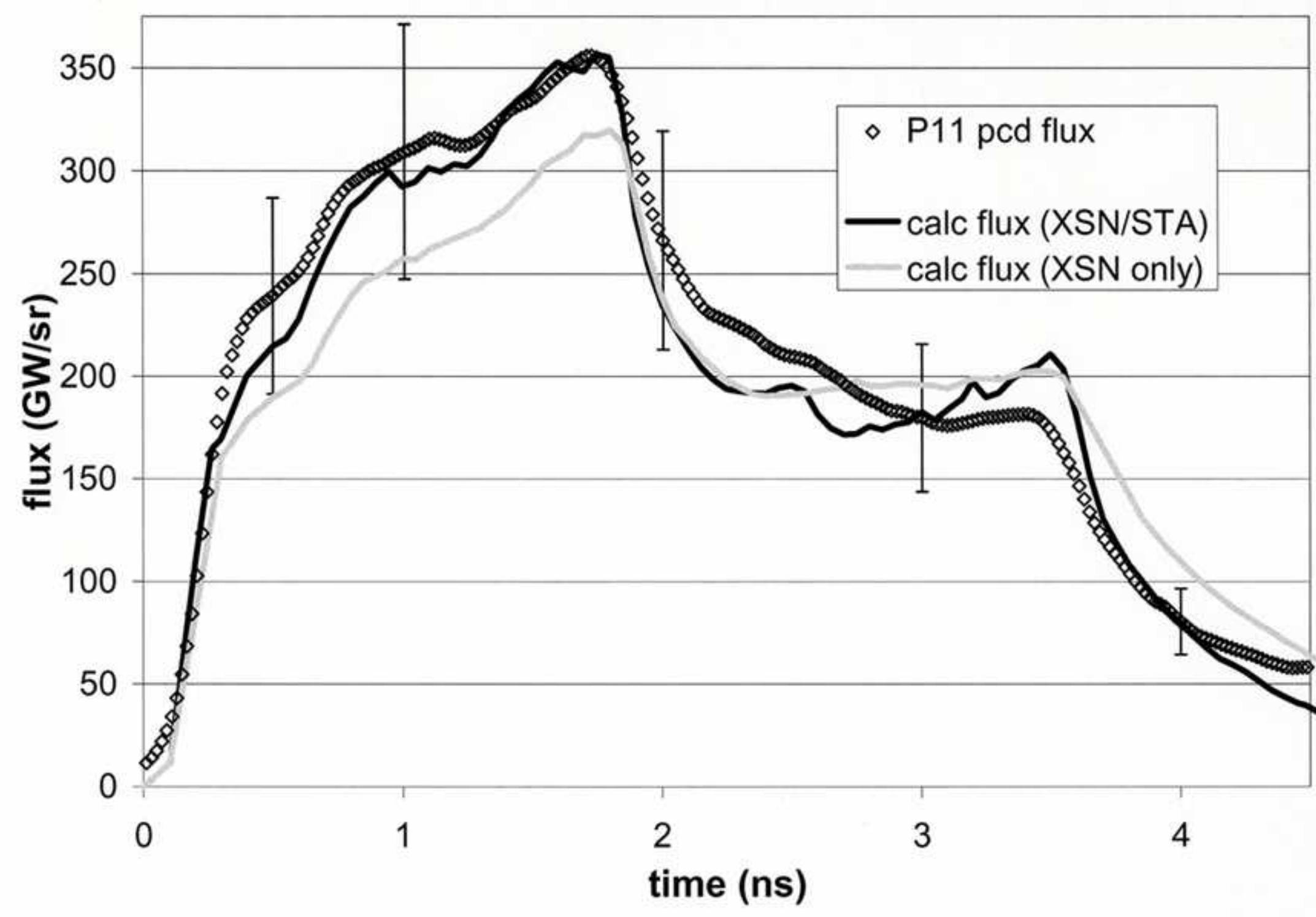


flux (GW/sr)

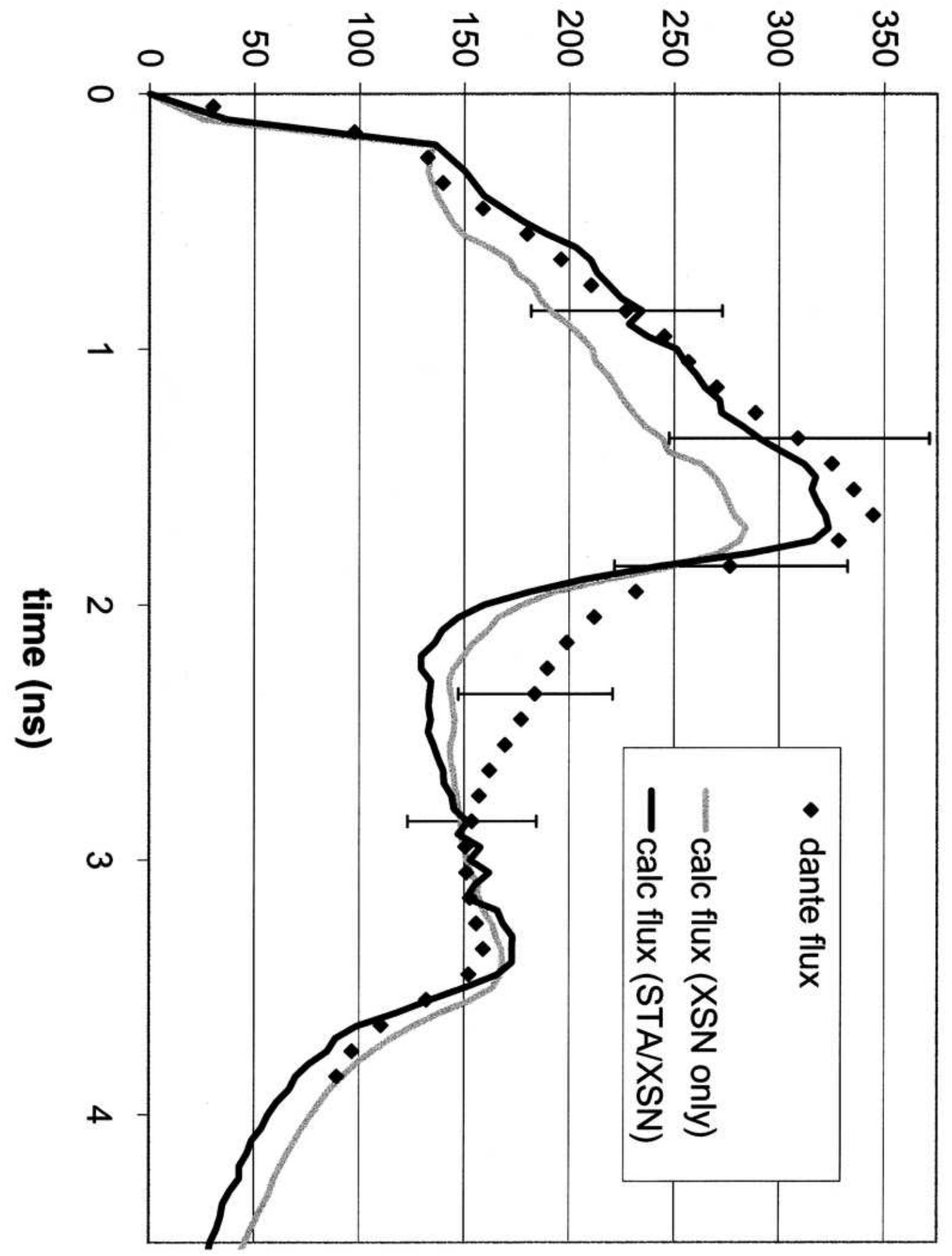




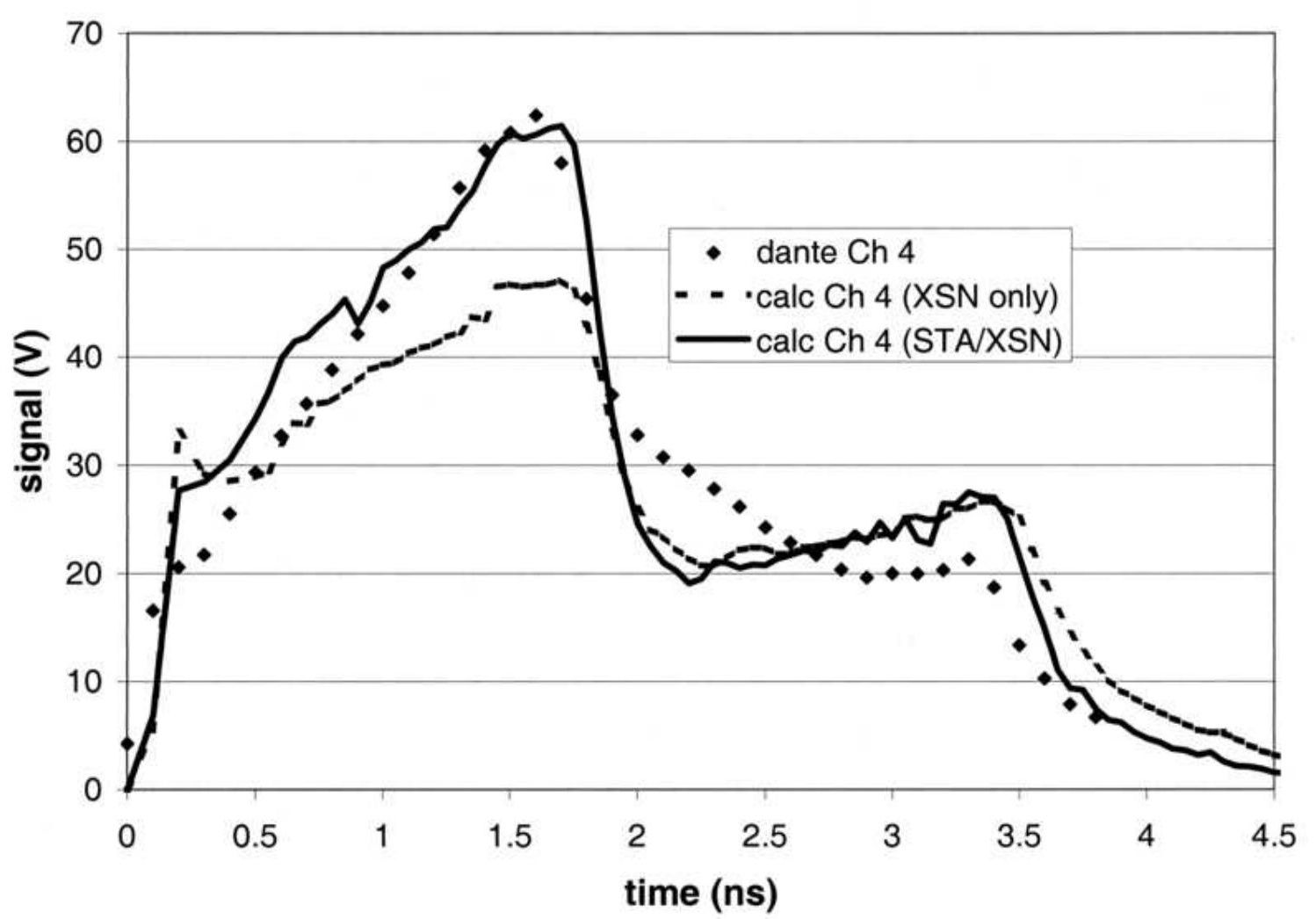




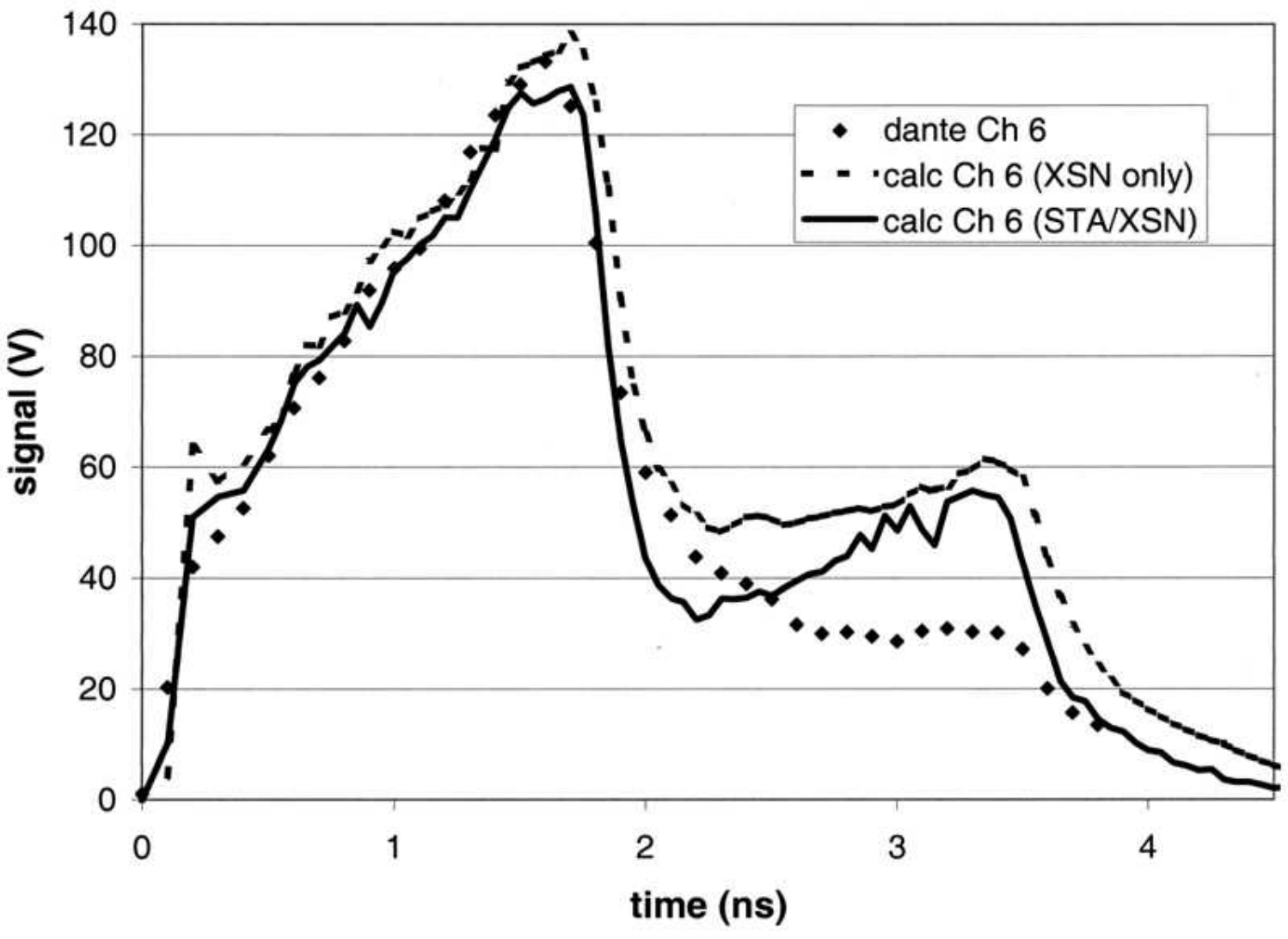




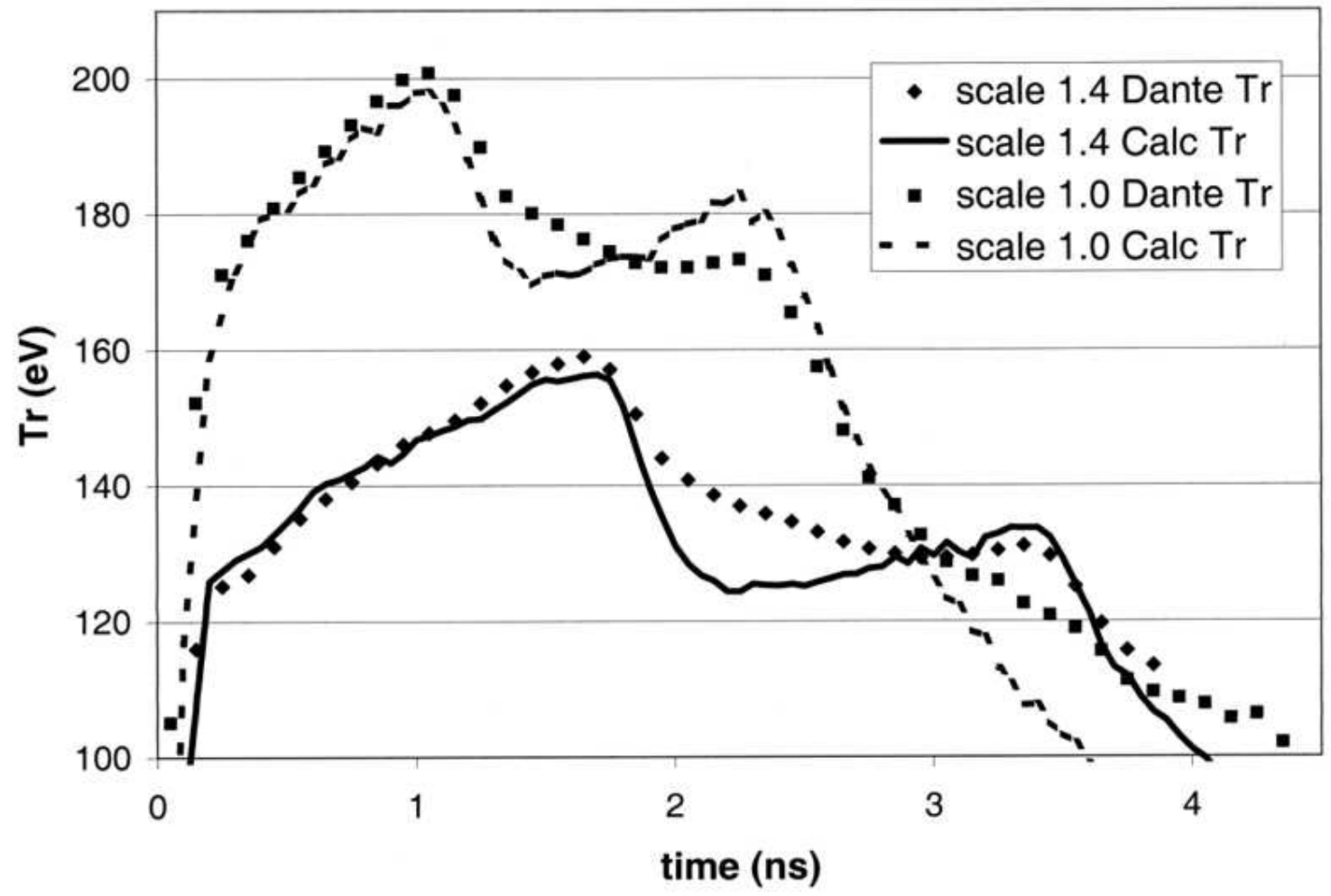




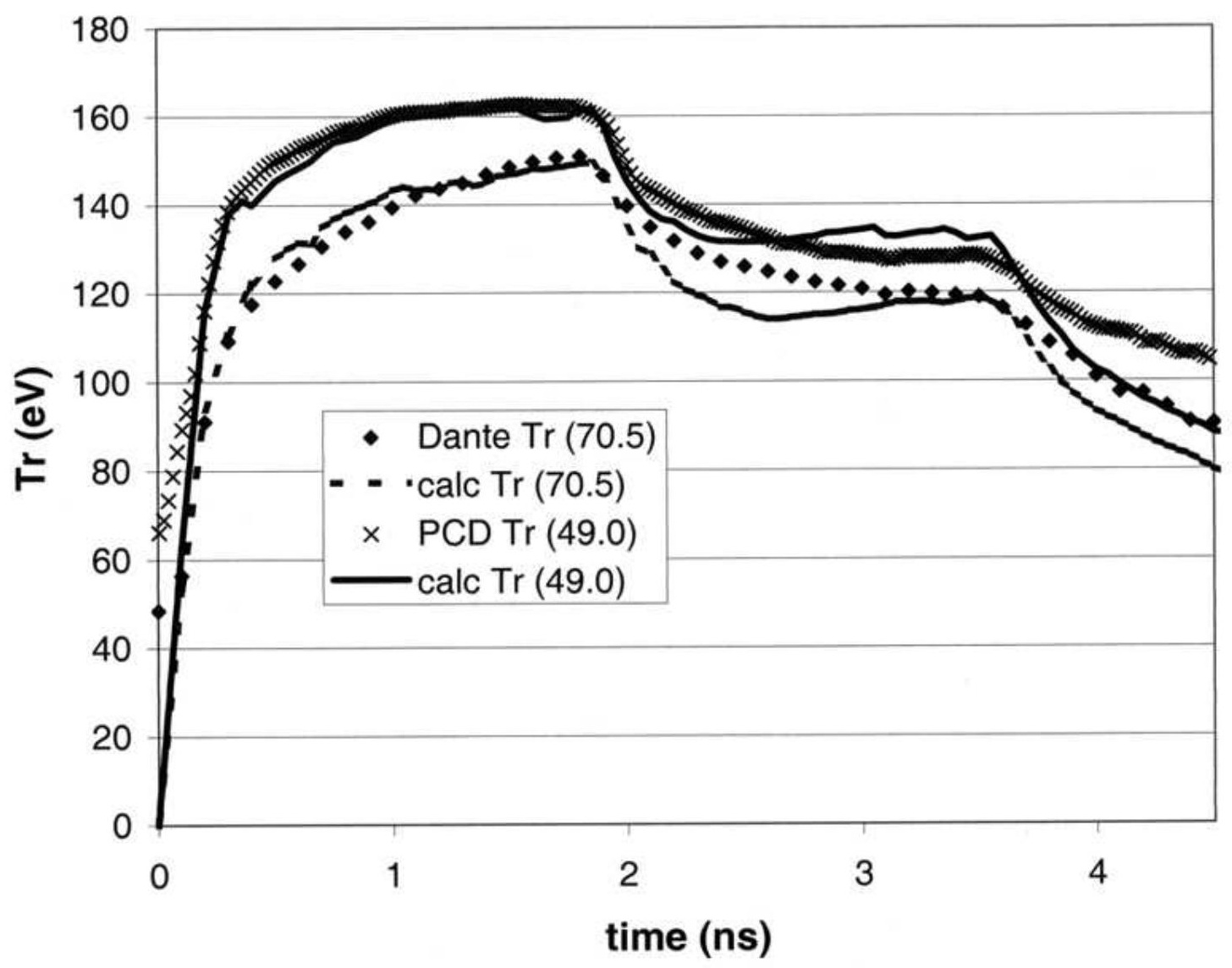

